\title{
HEAVY-FLAVOR PHOTO- AND ELECTROPRODUCTION AT NLO
}

\author{
I. SCHIENBEIN \\ Southern Methodist University \\ Department of Physics \\ 104 Fondren Science Building \\ 3215 Daniel Avenue \\ Dallas, Texas 75275-0175, USA \\ E-mail: schien@physics.smu.edu
}

\begin{abstract}
We review one-particle inclusive production of heavy-flavored hadrons in a framework which resums the large collinear logarithms through the evolution of the FFs and PDFs and, at the same time, retains the full dependence on the heavy-quark mass without additional theoretical assumptions. The main focus is on the production of $D^{\star}$ mesons in deep inelastic electron-proton scattering at HERA. We show results, neglecting for the time being the heavy-quark mass terms, for deep inelastic $D^{\star}$ meson production at finite transverse momenta. Work to implement this process in the above mentioned massive QCD framework is in progress.
\end{abstract}

\section{Introduction}

One-particle inclusive production processes provide extensive tests of perturbative quantum chromodynamics (pQCD). In contrast to fully inclusive processes, it is possible to study distributions in the momentum of the final state particle and to apply kinematical cuts which come close to the experimental situation. On the other hand, contrary to even more exclusive cases, QCD factorization theorems ${ }^{1,2}$ still hold stating that this class of observables can be computed as convolutions of universal parton distribution functions (PDFs) and fragmentation functions (FFs) with perturbatively calculable hard scattering cross sections. As is well-known, it is due to the factorization property that the parton model of QCD has predictive power. Hence, tests of the universality of the PDFs and FFs are of crucial importance for validating this QCD framework. At the same time, lowest order expressions for the hard scattering cross sections are often not sufficient for meaningful tests and the use of higher order computations is mandatory.

The perturbative analysis is becoming more involved and interesting 
if the observed final state hadron contains a heavy (charm or bottom) quark. In this case, the heavy-quark mass $m_{h}$ enters as an additional scale. Clearly, the conventional massless formalism, also known as zeromass variable-flavor-number scheme (ZM-VFNS), can also be applied to this case, provided the hard scale $Q$ of the process is much bigger than the heavy-quark mass such that terms $m_{h} / Q$ are negligible. However, at present collider energies, most of the experimental data lie in the kinematic region $Q \gtrsim m_{h}$ and it is necessary to take the power-like mass terms into account in a consistent framework.

The subject of this article is the theoretical description of one-particle inclusive production of heavy-flavored hadrons $X_{h}=D, B, \Lambda_{c}, \ldots$ in a massive variable-flavor-number scheme (GM-VFNS). In such a scheme the large collinear logarithms of the heavy-quark mass $\ln \mu / m_{h}$ are subtracted from the hard scattering cross sections and resummed through the evolution of the fragmentation functions (FFs) and parton distribution functions (PDFs). At the same time, finite non-logarithmic mass terms $m_{h} / Q$ are kept in the hard part and fully taken into account.

In order to test the pQCD formalism, in particular the universality of the FFs, it is important to provide a description of all relevant processes in a coherent framework. Therefore, it is important to work out the GMVFNS at next-to-leading order (NLO) of QCD for all the relevant processes. Previously, the GM-VFNS has been applied to the following processes: $\gamma+\gamma \rightarrow D^{\star+}+X$ (direct part) ${ }^{3}, \gamma+\gamma \rightarrow D^{\star+}+X$ (single resolved part) ${ }^{4}, \gamma+p \rightarrow D^{\star+}+X$ (direct part) ${ }^{5}, p+\bar{p} \rightarrow\left(D^{0}, D^{\star+}, D^{+}, D_{s}^{+}\right)+X$

$6,7,8$, where the latter results for hadron-hadron collisions also constitute the resolved contribution to the photoproduction process $\gamma+p \rightarrow X_{h}+X$.

In this contribution, we will review the production of heavy-flavored hadrons $X_{h}$ in hadron-hadron, photon-proton and deep inelastic electronproton collisions where the main focus will be on the electroproduction case. We will show results, neglecting for the time being the $m_{h} / Q$ mass terms, for deep inelastic $D^{\star}$ meson production at finite transverse momenta.

\section{Theoretical Framework}

\subsection{GM-VFNS}

The differential cross sections for inclusive heavy-flavored hadron production can be computed in the GM-VFNS according to the familiar factorization formulae, however, with heavy-quark mass terms included in the hard scattering cross sections ${ }^{9}$. Generically, the physical cross sections are 
expressed as convolutions of PDFs for the incoming hadron(s), hard scattering cross sections, and FFs for the fragmentation of the outgoing parton into the observed hadron. All possible partonic subprocesses are taken into account. The massive hard scattering cross sections are constructed in a way that in the limit $m_{h} \rightarrow 0$ the conventional ZM-VFNS is recovered. A more detailed discussion of the GM-VFNS and the construction of the massive hard scattering cross sections can be found in Refs. 6, 7 and the conference proceedings ${ }^{10,11}$.

\subsection{Fragmentation Functions}

A crucial ingredient entering these calculation are the non-perturbative FFs for the transition of the final state parton into the observed hadron $X_{h}$. For charm-flavored mesons, $X_{c}$, three sets of FFs have been employed in our analyses: (i) BKK98-D ${ }^{12}$ : For $X_{c}=D^{*+}$, such FFs were extracted at LO and NLO in the $\overline{\mathrm{MS}}$ factorization scheme with $n_{f}=5$ massless quark flavors several years ago ${ }^{12}$ from the scaled-energy $(x)$ distribution $d \sigma / d x$ of the cross section of $e^{+} e^{-} \rightarrow D^{*+}+X$ measured by the ALEPH ${ }^{13}$ and OPAL ${ }^{14}$ Collaborations at CERN LEP1. (ii) KK05-D ${ }^{15}$ : Recently, the BKK98$\mathrm{D}$ analysis was extended ${ }^{15}$ to include $X_{c}=D^{0}, D^{+}, D_{s}^{+}, \Lambda_{c}^{+}$by exploiting appropriate OPAL data ${ }^{16}$. (iii) KK05-D2: In Refs. ${ }^{12,15}$, the starting scales $\mu_{0}$ for the DGLAP evolution of the $a \rightarrow X_{c}$ FFs in the factorization scale $\mu_{F}^{\prime}$ have been taken to be $\mu_{0}=2 m_{c}$ for $a=g, u, \bar{u}, d, \bar{d}, s, \bar{s}, c, \bar{c}$ and $\mu_{0}=2 m_{b}$, with $m_{b}=5 \mathrm{GeV}$, for $a=b, \bar{b}$. The FFs for $a=g, u, \bar{u}, d, \bar{d}, s, \bar{s}$ were assumed to be zero at $\mu_{F}^{\prime}=\mu_{0}$ and were generated through the DGLAP evolution to larger values of $\mu_{F}^{\prime}$. For consistency with the $\overline{\mathrm{MS}}$ prescription for PDFs, we repeated the fits of the $X_{c} \mathrm{FFs}$ for the choice $\mu_{0}=m_{c}, m_{b}$. This changes the $c$-quark FFs only marginally, but has an appreciable effect on the gluon FF, which is important at Tevatron energies, as was found for $D^{*+}$ production in Ref. ${ }^{6}$. These new FFs will be presented elsewhere.

\subsection{Input Parameters}

If not stated otherwise, the following parameters have been chosen for the numerical results presented below. For the proton PDFs we have employed the CTEQ6M/CTEQ6.1M PDFs from the CTEQ Collaboration ${ }^{17}$ and the KK05-D2 FFs. We have set $m_{c}=1.5 \mathrm{GeV}, m_{b}=5 \mathrm{GeV}$ and have used the two-loop formula for $\alpha_{s}^{\left(n_{f}\right)}\left(\mu_{R}\right)$ in the $\overline{\mathrm{MS}}$ scheme with $\alpha_{s}^{(5)}\left(m_{Z}\right)=0.118$. Our theoretical predictions depend on three scales, the renormalization scale $\mu_{R}$, and the initial- and final-state factorization scales $\mu_{F}$ and $\mu_{F}^{\prime}$, 

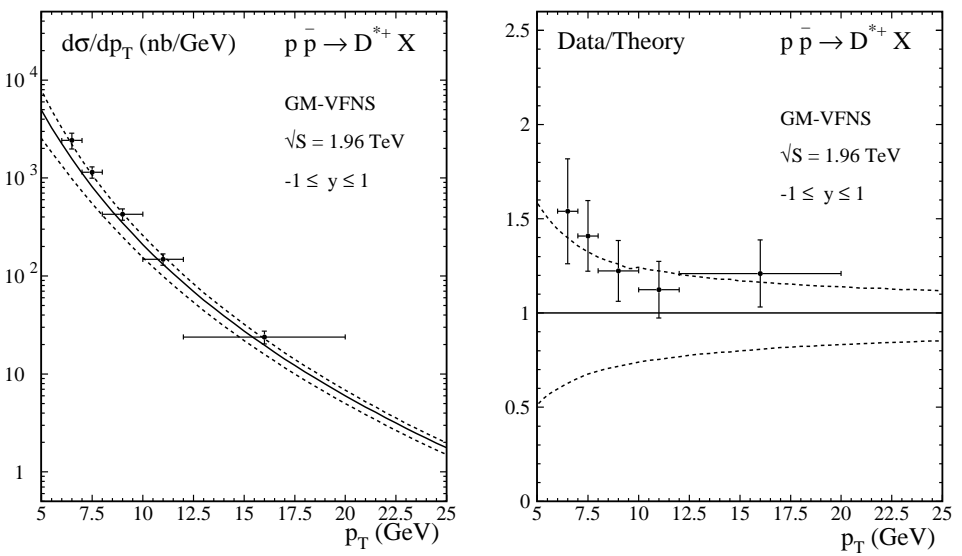

Figure 1. Comparison of the CDF data ${ }^{18}$ with our NLO predictions for $D^{*+}$. The solid line represents our default prediction obtained with $\mu_{R}=\mu_{F}=\mu_{F}^{\prime}=m_{T}$, while the dashed lines indicate the scale uncertainty estimated by varying $\mu_{R}, \mu_{F}$, and $\mu_{F}^{\prime}$ independently within a factor of 2 up and down relative to the central values. The right figure shows the data-over-theory representation with respect to our default prediction.

respectively. Our default choice for hadro- and photoproduction has been $\mu_{R}=\mu_{F}=\mu_{F}^{\prime}=m_{T}$, where $m_{T}=\sqrt{p_{T}^{2}+m_{h}^{2}}$ is the transverse mass. The scale choice in the electroproduction case will be specified below.

\section{Hadroproduction}

Recently, the CDF collaboration has published first cross section data for inclusive production of $D^{0}, D^{+}, D^{*+}$, and $D_{s}^{+}$mesons in $p \bar{p}$ collisions ${ }^{18}$ obtained in Run II at the Tevatron at center-of-mass energies of $\sqrt{S}=1.96$ $\mathrm{TeV}$. The data come as distributions $d \sigma / d p_{T}$ with $y$ integrated over the range $|y| \leq 1$ and the particle and antiparticle contributions are averaged.

Our theoretical predictions in the GM-VFNS are compared with the CDF data for $D^{\star}$ mesons on an absolute scale in Fig. 1 (left) and in the data-over-theory representation with respect to our default results in Fig. 1 (right). We find good agreement in the sense that the theoretical and experimental errors overlap where the experimental results are gathered on the upper side of the theoretical error band, corresponding to a small value of $\mu_{R}$ and large values of $\mu_{F}$ and $\mu_{F}^{\prime}$, the $\mu_{R}$ dependence being dominant in the upper $p_{T}$ range. As is evident from Fig. 1 (right), the central data points tend to overshoot the central QCD prediction by a factor of about 1.5 
at the lower end of the considered $p_{T}$ range, where the errors are largest, however. This factor is rapidly approaching unity as the value of $p_{T}$ is increased. The tendency of measurements of inclusive hadroproduction in Tevatron run II to prefer smaller renormalization scales is familiar from single jets, which actually favor $\mu_{R}=p_{T} / 2^{19}$.

For more details and a comparison with the data for the $D^{0}, D^{+}$, and $D_{s}^{+}$mesons we refer to Ref. 8. A comparison of NLO predictions for $p+\bar{p} \rightarrow$ $B^{+}+X$ in the GM-VFNS with recent CDF data ${ }^{20}$ is in preparation ${ }^{21}$.

\section{Photoproduction}

Inclusive photoproduction of $D^{\star}$ mesons, $\gamma+p \rightarrow D^{\star}+X$, has been studied in Ref. 5 where the direct part has been computed in the GM-VFNS whereas the resolved part has been included in the ZM-VFNS. In this analysis the BKK98-D FFs have been utilized and, for the resolved contribution, the GRV92 photon PDFs ${ }^{22}$. The other parameters have been chosen as specified in Sec. 2.3. In Fig. 6 of Ref. 5, the central numerical predictions for the transverse momentum $\left(p_{T}\right)$ distributions of the $D^{\star}$ meson have been compared with preliminary ZEUS data ${ }^{23}$. There exist similar data by the $\mathrm{H} 1$ collaboration ${ }^{24}$ which have not been used in this analysis. As can be seen in this figure, the agreement of the $p_{T}$-distributions with the data is quite good down to $p_{T} \simeq 2 m_{c}$ and the mass effects turn out to be small. In order to extend the range of applicability of the GM-VFNS into the region $p_{T}<3 \mathrm{GeV}$ more work on the matching to the 3 -fixed flavor theory would be needed. The Figs. 7 - 9 of Ref. 5, showing results for the rapidity $(y)$, invariant mass $(W)$ and inelasticity $\left(z\left(D^{\star}\right)\right)$ distributions, have to be taken with a grain of salt since they receive large contributions from the transverse momentum region $1.9<p_{T}<3 \mathrm{GeV}$ which is outside the range of validity of the present theory.

With the work in Ref. 6, it is now possible to include also the resolved part in the GM-VFNS. It will be interesting to compare the complete GMVFNS framework at NLO of QCD, combined with updated FFs, in more detail with ZEUS and $\mathrm{H} 1$ photoproduction data once they are finalized.

\section{Electroproduction}

Recently, the single inclusive electroproduction of light hadrons at finite transverse momenta has attracted quite a lot of interest, where the outgoing hadron is required to carry a non-vanishing transverse momentum $\left(p_{T}^{*}\right)$ in the center-of-mass system (CMS) of the virtual photon and the incoming 
proton. The following partonic subprocesses contribute at leading order (LO) and are of the order $O\left(\alpha_{s}\right): \gamma^{\star}+q \rightarrow q+g$ and $\gamma^{\star}+g \rightarrow q+$ $\bar{q}$. Very recently, the NLO $\left(O\left(\alpha_{s}^{2}\right)\right)$ corrections to this process have been accomplished by three independent groups ${ }^{25,26,27}$. Suffice to say here that the NLO corrections increase the LO results in certain kinematical regions by large factors and are essential for bringing theory in agreement with the experimental results. For more details see Ref. 28.

Endowed with appropriate fragmentation functions, the computation in Ref. 27 has been employed to obtain predictions in the ZM-VFNS for the production of heavy-flavored hadrons in electron-proton collisions at HERA, $e+p \rightarrow e+X_{h}+X$. The electron and proton energies have been set to $E_{e}=27.5 \mathrm{GeV}$ and $E_{p}=820 \mathrm{GeV}$ (Fig. 2) resp. $920 \mathrm{GeV}$ (Figs. 3, 4) in the laboratory frame. Furthermore, the renormalization and factorization scales have been chosen ${ }^{27}$ as $\mu_{R}^{2}=\mu_{F}^{2}=\mu_{F}^{\prime 2}=\xi \frac{Q^{2}+\left(p_{T}^{*}\right)^{2}}{2}$. The dimensionless parameter $\xi$ has been varied between $1 / 2$ and 2 about the default value 1 in order to estimate the scale uncertainties of the theoretical predictions. The other input parameters have been chosen as described in Sec. 2.3. The following cuts have been imposed on the numerical results in Figs. $2-4: 2<Q^{2}<100 \mathrm{GeV}^{2}, 0.05<y<0.7,1.5 \leq p_{T, \mathrm{Lab}}\left(D^{\star}\right) \leq 15 \mathrm{GeV}$, and $\left|\eta_{\text {Lab }}\left(D^{\star}\right)\right|<1.5$ where the momentum transfer $Q^{2}$, the inelasticity $y$, and the pseudorapidity $\eta$ are defined as usual. Furthermore, in Figs. 3-4 we have asked for the additional constraint $p_{T}^{*}\left(D^{\star}\right)>2 \mathrm{GeV}$ where $p_{T}^{*}\left(D^{\star}\right)$ is the transverse momentum of the $D^{\star}$ meson in the $\gamma^{*} p$-CMS. This cut is essential to avoid the collinear singularities as $p_{T}^{*} \rightarrow 0$. The results have been calculated with $n_{f}=5$ flavors and include the contribution where a bottom quark fragments into the $D^{\star}$ meson via $b \rightarrow B \rightarrow D^{\star}$. No distinction is made between $D^{\star+}$ and $D^{\star-}$. Figure 2 shows the $p_{T}^{*}$ spectrum in comparison with $\mathrm{H} 1$ data ${ }^{29}$, collected in the years 1994 to 1996 with $27.5 \mathrm{GeV}$ positrons colliding with $820 \mathrm{GeV}$ protons at CMS energies of $\sqrt{S}=300 \mathrm{GeV}$. The data points are reasonably well described if one keeps in mind that the theory is expected to work for larger $p_{T}^{*} \gtrsim 2 \mathrm{GeV}$. Furthermore, in the ZM-VFNS the heavy-quark mass terms are missing which are potentially important in the region of small $p_{T}^{*}$. These terms will be included as soon as ongoing work to implement this process in the GM-VFNS has been completed.

Finally, results for other distributions $\left(p_{T, \mathrm{Lab}}, \eta_{\mathrm{Lab}}, W, z, Q^{2}\right.$, and $\left.x_{\mathrm{Bj}}\right)$ are presented in Figs. 3-4. 


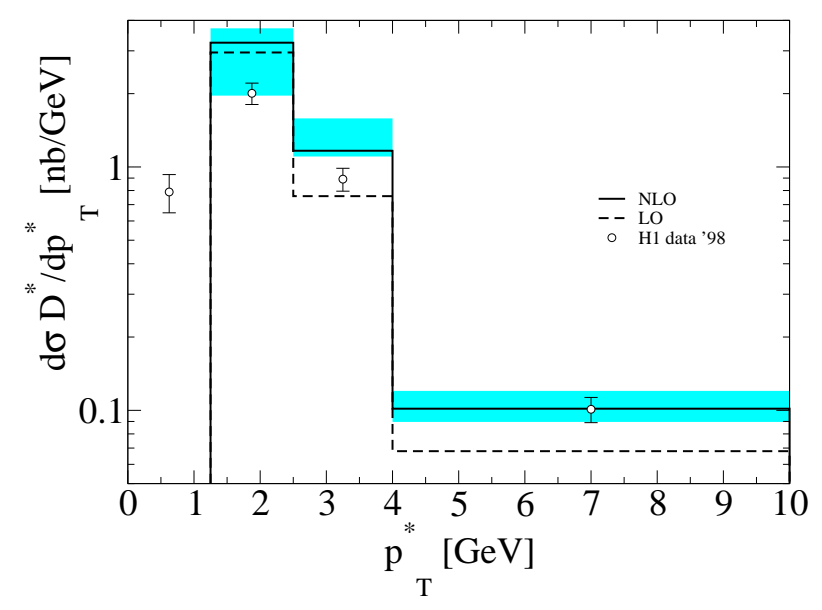

Figure 2. NLO QCD predictions in the ZM-VFNS for electroproduction of $D^{\star}$ mesons in dependence of the transverse momentum of the $D^{\star}$ meson in the $\gamma^{\star} p$ CMS. H1 data 29 are shown for comparison. See the text for further details.
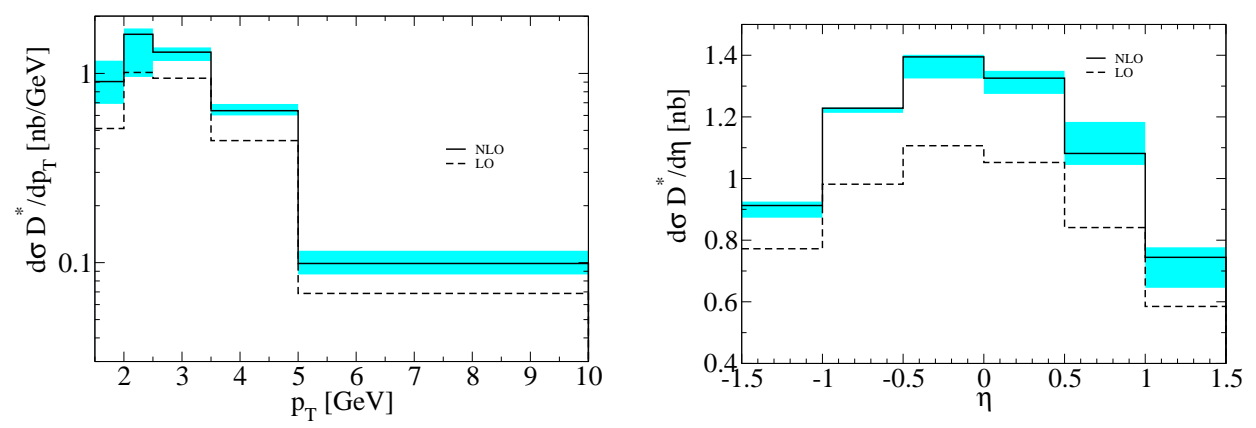

Figure 3. NLO QCD predictions in the ZM-VFNS for electroproduction of $D^{\star}$ mesons in dependence of the transverse momentum (left) and the pseudo-rapidity (right) of the $D^{\star}$ meson in the laboratory frame. For further details on the kinematical cuts and the uncertainty band see the text.

\section{Summary}

We have discussed one-particle inclusive production of heavy-flavored hadrons in hadron-hadron, photon-proton, and electron-proton collisions in a massive variable-flavor-number scheme (GM-VFNS). The importance of a unified treatment of all these processes, based on QCD factorization theorems, has been emphasized, in order to provide meaningful tests of the 

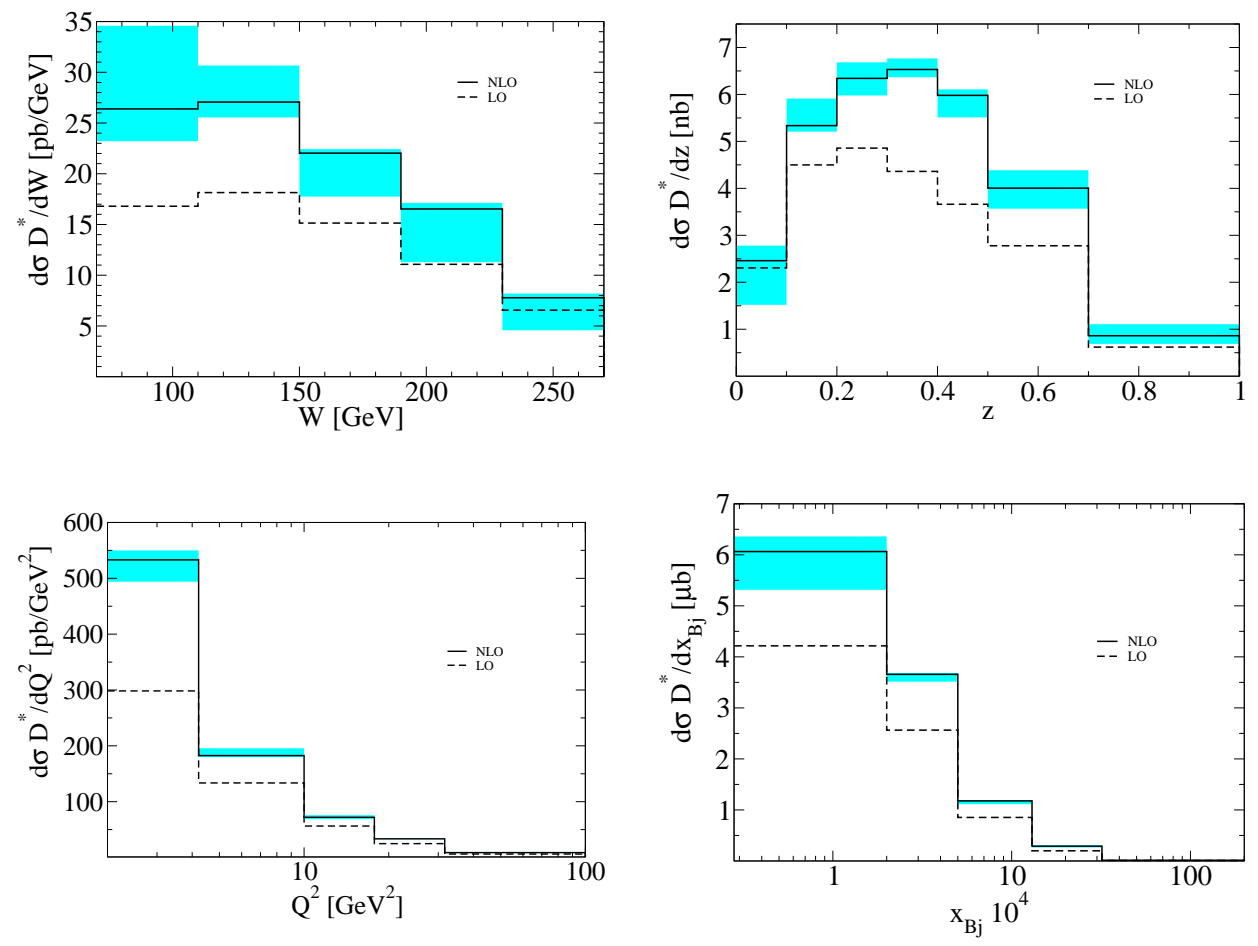

Figure 4. As in Fig. 3 for the $\gamma^{\star} p$ invariant mass $W$, the inelasticity $z$, the momentum transfer $Q^{2}$ and Bjorken- $x$.

universality of the FFs and hence of QCD. At the same time, it is necessary to incorporate heavy-quark mass effects in the formalism since many of the present experimental data points lie in a kinematical region where the hard scale of the process is not much larger than the heavy-quark mass. This is achieved in the GM-VFNS, which includes heavy-quark mass effects in a rigorous way and still relies on QCD factorization. We have discussed numerical results for the three reactions. In general, the description of the transverse momentum spectra is quite good down to transverse momenta $p_{T} \simeq 2 m_{h}$. Extending the range of applicability of our scheme to smaller $p_{T}$ would require more work on the matching to the corresponding theories in the fixed flavor number schemes. Our ZM-VFNS results for the electroproduction of $D^{\star}$ mesons indicate that future experimental results by the H1 collaboration can be nicely described. All this leads us to the expectation that a good overall description of the data can be reached in the future 


\section{Acknowledgments}

The author would like to thank the organizers of the Ringberg workshop on New Trends in HERA Physics 2005 for the kind invitation, B. A. Kniehl, G. Kramer and H. Spiesberger for their collaboration, and M. Maniatis for providing the figures $2-4$ and for useful discussions.

\section{References}

1. J. C. Collins, D. E. Soper, and G. Sterman, in Perturbative Quantum Chromodynamics, edited by A. H. Mueller (World Scientific, 1989).

2. J. C. Collins and D. E. Soper, Ann. Rev. Nucl. Part. Sci. 37, 383 (1987).

3. G. Kramer and H. Spiesberger, Eur. Phys. J. C22, 289 (2001).

4. G. Kramer and H. Spiesberger, Eur. Phys. J. C28, 495 (2003).

5. G. Kramer and H. Spiesberger, Eur. Phys. J. C38, 309 (2004).

6. B. A. Kniehl, G. Kramer, I. Schienbein, and H. Spiesberger, Phys. Rev. D71, 014018 (2005).

7. B. A. Kniehl, G. Kramer, I. Schienbein, and H. Spiesberger, Eur. Phys. J. C41, 199 (2005).

8. B. A. Kniehl, G. Kramer, I. Schienbein, and H. Spiesberger, Phys. Rev. Lett. 96, 012001 (2006).

9. J. C. Collins, Phys. Rev. D58, 094002 (1998).

10. I. Schienbein, Open heavy-flavour photoproduction at NLO, Proceedings of the Ringberg Workshop, New Trends in HERA Physics 2003, edited by G. Grindhammer, B. A. Kniehl, G. Kramer and W. Ochs, World Scientific, 2004, p. 197; I. Schienbein, Proceedings of the 12th International Workshop on Deep Inelastic Scattering (DIS 2004), Strbske Pleso, Slovakia, 14-18 Apr 2004, hep-ph/0408036; B. A. Kniehl, G. Kramer, I. Schienbein, and H. Spiesberger, Proceedings of the 13th International Workshop on Deep Inelastic Scattering (DIS 2005), Madison, Wisconsin, USA, April 27 - May 1,2005, hep-ph/0507068.

11. J. Baines et al, Summary report of the heavy-flavour working group of the HERA-LHC Workshop, hep-ph/0601164.

12. J. Binnewies, B. A. Kniehl, and G. Kramer, Phys. Rev. D58, 014014 (1998).

13. R. Barate et al., ALEPH Collaboration, Eur. Phys. J. C16, 597 (2000).

14. K. Ackerstaff et al., OPAL Collaboration, Eur. Phys. J. C1, 439 (1998).

15. B. A. Kniehl and G. Kramer, Phys. Rev. D71, 094013 (2005).

16. G. Alexander et al., OPAL Collaboration, Z. Phys. C72, 1 (1996).

17. J. Pumplin et al., JHEP 07, 012 (2002); D. Stump et al., JHEP 10, 046 (2003).

18. D. Acosta et al., CDF Collaboration, Phys. Rev. Lett. 91, 241804 (2003).

19. R. Field, for the CDF Collaboration, in Proceedings of the XIII ${ }^{\text {th }}$ International Workshop on Deep Inelastic Scattering (DIS05), Madison, Wisconsin, 2005 (American Institute of Physics, Melville, to be published); B. Davies, for the D0 Collaboration, ibid.

20. D. Acosta et al., CDF Collaboration, Phys. Rev. D71, 032001 (2005). 
21. B. A. Kniehl, G. Kramer, I. Schienbein, and H. Spiesberger, (work in progress).

22. M. Glück, E. Reya, and A. Vogt, Phys. Rev. D46, 1973 (1992).

23. ZEUS Collaboration, S. Chekanov et al., 31st International Conference on High Energy Physics, ICHEP02, July 24-31 2002, Amsterdam, Abstract 786; see also: ZEUS Collaboration, http://www-zeus.de/public_plots, Measurement of $D^{\star}$ photoproduction at HERA, 2003.

24. H1 Collaboration, International Europhysics Conference on High Energy Physics, EPS03, July 17-23 2003, Aachen, Abstract 097, DESY-H1prelim03-071 and earlier H1 paper given in this reference.

25. P. Aurenche, R. Basu, M. Fontannaz, and R. M. Godbole, Eur. Phys. J. C34, 277 (2004).

26. A. Daleo, D. de Florian, and R. Sassot, Phys. Rev. D71, 034013 (2005).

27. B. A. Kniehl, G. Kramer, and M. Maniatis, Nucl. Phys. B711, 345 (2005); Erratum-ibid. B720(2005)345.

28. B. A. Kniehl, these proceedings.

29. C. Adloff et al., H1 Collaboration, Nucl. Phys. B545, 21 (1999). 